\section{An Improved Forward $I-V$ Method for Nonideal Schottly Diodes with High Series Resistance}

\section{C.-D. LIEN, F. C. T. SO, AND M.-A. NICOLET}

\begin{abstract}
Two methods are described to obtain the value of the series resistance $(R)$ of a Schottky diode from its forward $I-V$ character istic. The value of $R$ is then used to plot the curve $\ln (I)$ versus $V_{D}(=V \ldots I R)$ which becomes a straight line even if $\ln (I)$ versus $V$ does not. The ideality factor $n$ and the Schottky-barrier height $\phi_{B O}$ of the diode then follow from the standard procedure. The main advantages of the methods are: 1) a linear regression can be used to calculate the valie of $R, 2)$ many data points are used over the whole data range which rutises the accuracy of the results, and 3 ) the validity of constant $R$ assumption can be checked by the linearity of the $\ln (I)$ versus $V_{D}$ curve. The methods are illustrated on the experimental data of a real diode.
\end{abstract}

\section{INTRODUCTION}

For a real Schottky diode, the $I-V$ characteristic (see, e.g., Fig. 1) is often approximated by

$$
I=I_{s}\left(\exp \left(\frac{\beta V_{D}}{n}\right)-1\right) \approx I_{s} \exp \left(\frac{\beta V_{D}}{n}\right)
$$

(for $\beta V_{D} \gg 1$ ), ${ }^{1}$ where $n$ is the ideality factor, $V_{D}$ is the visltage across the diode, $\beta=q / k T$, and

$$
I_{s}=A A^{* *} T^{2} \exp \left(-\beta \phi_{B 0}\right)=I_{0} \exp \left(-\beta \phi_{B 0}\right)
$$

and the applied voltage $V$ is assumed to differ from $V_{D}$ b: a value $I R$ (i.e., $V_{D}=V-I R$ ), where $R$ is defined as the seives resistance of the diode. A well-established method of determining the barrier height $\phi_{B 0}$ and the ideality factor $n$ is to make a $\ln (I)$ versus $V$ plot. For voltages larger than several $k T / q$ and small $R$, this plot will be a straight line whose slcpe and extrapolated intercept with the zero voltage axis can be used to calculate the ideality factor and the barrier heig,ht (standard $I-V$ method).

As the series resistance increases, the linear region in the ln il) versus $V$ plot shrinks resulting in an inaccurate measurement of $n$ and $\phi_{B 0}$. To remedy this difficulty, Norde [1] has p:oposed a modified method for the case of "a Schottky diode with $n=1$ by finding the minimum of the function

$$
F(V)=0.5 \mathrm{~V}-\frac{1}{\beta} \ln \left(\frac{I(V)}{I_{0}}\right) .
$$

The disadvantages of the Norde method are that 1) the ideality factor is assumed to be unity, which is not always true for a real diode, and 2) a few data points near the minimum of $F(V)$ are actually used to calculate the barrier height. This latter fact tends to increase the statistical error of the result.

Here we suggest two methods to obtain the value of $R$ and

Manuscript received April 20, 1984. This work was sponsored in part by the Jet Propulsion Laboratory, California Institute of Teshnology, Director's Discretionary Fund, through an agreement w:th the National Aeronautics and Space Administration (A. Morrison).

The authors are with the Department of Electrical Engineering, Criifornia Institute of Technology, Pasadena, CA 91125.

${ }^{1}$ See, e.g., S. M. Sze, Physics of Semiconductor Devices, 2nd $\mathrm{d}$. New York: Wiley, 1982.

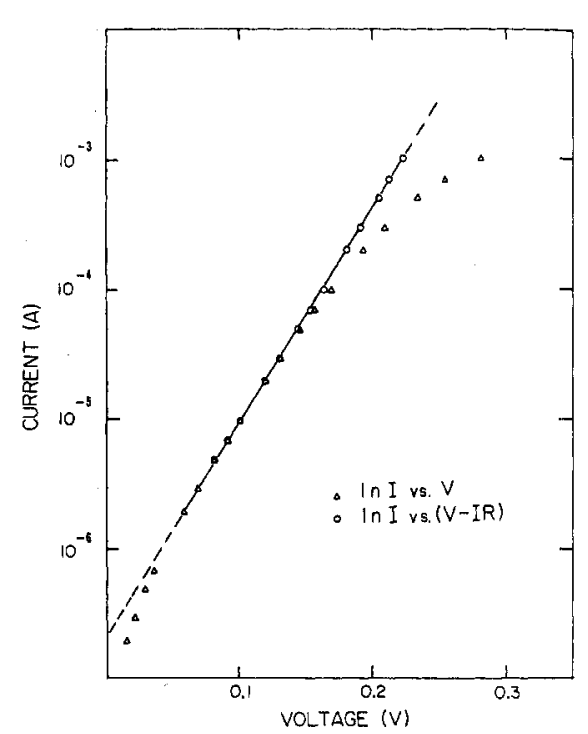

Fig. 1. Experimental data of $\ln (I)$-versus $V$ (triangles) for a $\mathrm{Ni}_{36} \mathrm{~W}_{64} / \mathrm{Si}$ Schottky diode on an n-type (100) Si. $T=20^{\circ} \mathrm{C}, A^{* *}=112 \mathrm{~A} \cdot \mathrm{K}^{-1}$. $\mathrm{cm}^{-2}$, and $A=1.97 \times 10^{-3} \mathrm{~cm}^{2}$ are used here. Using $R=58.3 \Omega$, we have plotted the $\ln (I)$ versus $V_{D}(=V-I R)$ (circles) curve which yields $n=1.05$, and $\phi_{B 0}=0.63 \mathrm{~V}$.

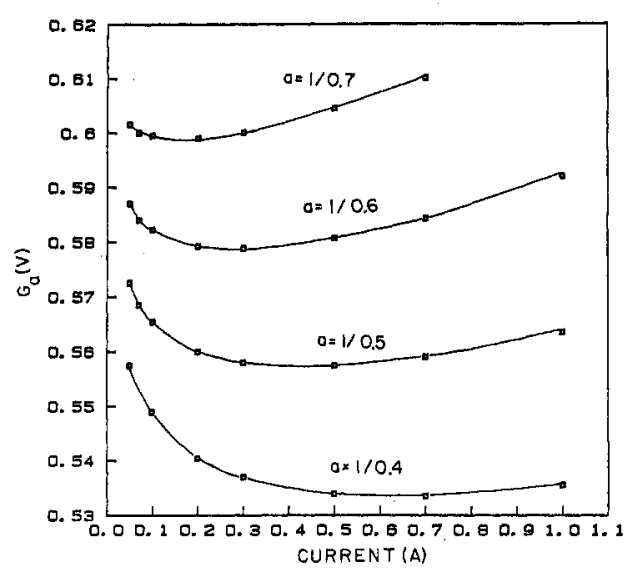

Fig. 2. $G_{a}(I)$, calculated from the $I-V$ data shown in Fig. 1, are plotted against $I$ for several $a$ 's. For each $a, G_{a}(I)$ has a minimum at $I=I_{a}$.

to use this $R$ to plot the $\ln (I)$ versus $V_{D}$ curve. The standard $I-V$ method is then used to obtain the values of $n$ and $\phi_{B O}$.

\section{METHOD}

The first method used to obtain the series resistance by plotting several Norde-type functions

$$
F_{a}(V)=V / a-\frac{1}{\beta} \ln \left(\frac{I(V)}{I_{0}}\right)
$$

versus $I$ (for $\beta V_{D} \gg 1$ ) and finding the minimum point of each $F_{a}$ for several $a$ 's, given ranges from $n$ (ideality factor) to $\infty$ (see e.g., Fig. 2). Since $V$ is a strictly monotonic function of $I, F_{a}$ can also be viewed as a function of $I$, viz., $G_{a}(I)=F_{a}(V)$. 


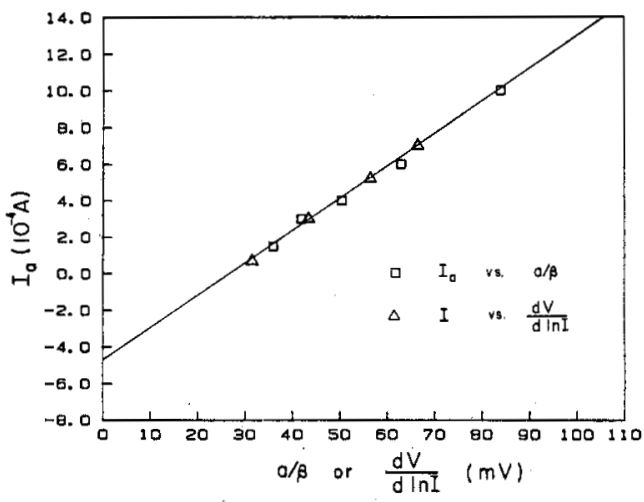

Fig. 3. $I_{a}$; obtained from Fig. 2, are plotted against $a / \beta$ (squares). The triangles are the data calculated from the sacond method as discussed in the text, i.e., $I$ versus $d V / d$ ln $(I)$. The calculated resistance value $R$ is equal to $58.3 \Omega$.

It turns out to be convenient to consider $G_{a}(I)$ rather than $F_{a}(V)$ because (4) contains $I$, not $V . G_{a}(I)$ has a minimum that (for each $a$ ranging from $n$ to $\infty$ ) occurs when $d G_{a}(I) / d I=0$, (assuming this occurred at $I=I_{a}$ ), i.e. ,

$$
\frac{d G_{a}\left(I_{a}\right)}{d I_{a}}=\frac{1}{a} \frac{d V_{a}}{d I_{a}}-\frac{1}{\beta I_{a}}=0 .
$$

This equation together with (1) results in

$$
I_{a}=\frac{1}{R \beta} a-\frac{n}{R \beta} \text {. }
$$

This equation shows that a plot of $I_{a}$ versus $a$ is a straight line (see e.g., Fig. 3) whose slope can be used to determine the value of $R$ and whose extrapolated intercept is at $I_{a}=0$ given $n$. The advantage of using a set of different values of $a$, rather than only one, resides in the fact that such a plot makes use of an extended range of data points of the $I-V$ characteristic. This plot also tests the assumption that the real diode can be modeled as a purely exponential characteristic diode in series with a constant resistance. In practice, if a linear relationship between $I_{a}$ and $a$ is observed, this plot is best used to obtain the value $R ; n$ is more accurately determined subsequently from the corrected $I-V$ characteristic.

The second method is based on (3), which states that $a^{-1}=$ $(1 / \beta) d \ln (I) / d V$ at $I=I_{a}$. This means that the parameter $a$ can be obtained for any given current $I=I_{a}$ by taking the derivatives of the measured $I-V$ characteristic at this point That procedure is easy to carry out on computerized $I-V$ plotters or with computer fitting of experimental data. An explicit evaluation of $G_{a}$ or $F_{a}$ as it is required in the Norde method is thus bypassed. With the parameter $a$ known for any value of $I_{a}$, the method again allows one to plot the relationship of (4) and derive the value of $R$. Obviously, mathematically the second method should yield the same $I_{a}$ versus $a$ plot as the first method.

Once the value of $R$ has been established by either method, its contribution to the experimental data points can be subtracted. After this subtraction, within experimental uncertainties, the characteristic should yield the straight line predicted by (1). This outcome means that the model of a purely exponential characteristic diode in series with a constant resistance is consistent with the data. Accurate values of $\Phi_{B 0}$ and $n$ can then be determined from this corrected characteristic.

As an example, Fig. 1 shows an experimental $I-V$ characteristic (triangles) of a Schottky diode plotted semi-logarithmi- cally. There is no linear region in this plot to allow for a reliable barrier height and ideality factor determination. Fig. 2 shows $G_{a}(I)$ versus $I$ curves for various $a$. The values of $I$ at the minimum points of $G_{a}$, defined as $I_{a}$, are then plotted against $a / \beta$ as shown in Fig. 3 (squares). The triangles shown in Fig. 3 are calculated by the second method, i.e., $I$ versus $d V / d \ln (I)$. The data derived from both methods agree very well. The fitted value of $R$ is $58.3 \Omega$. Using this value of $R$, we have plotted the $\ln (I)$ versus $V_{D}(=V-I R)$ data (circles) shown in Fig. 1. The ideality factor and barrier height are then determined by the standard method, which yields $n=1.05$ and $\phi_{B 0}=0.63 \mathrm{~V}$. The linearity of the plots in Figs. 1 and 3 shows that the assumption of a constant series resistance and a purely exponential characteristic diode is good over the range of operating points considered.

\section{CONCLUSION}

We have proposed an improved forward $I-V$ method to calculate the series resistance, ideality factor, and barrier height of a Schottky diode. Applying this method to experimental data of a real diode, we obtain quite a good result.

\section{ACKNOWLEDGMENT}

The authors acknowledge T. Banwell for helpful discussions.

\section{REFERENCES}

[1] H. Norde, "A modified $I-V$ plot for Schottky diodes with high series resistance," J. Appl. Phys., vol. 50, no. 7, pp. 5052-5053, 1979.

\section{Temperature Dependence of the Nonideal Component of Base Current in Micropower n-p-n Transistors}

\section{CARLOS GONZALEZ-BRIS AND ELIAS MUÑOZ}

Abstract-The low-temperature dependence of the nonideal base current component has been studied in low-concentration emitter n-p-n $\mathrm{Si}$ transistors. Under forward bias, below a certain temperature, ideality factors greater than 2 have been found and $h_{F E}$ roll-off characteristics deviate from a S-R-H recombination model.

\section{INTRODUCTION}

The behavior of n-p-n BJT's at very low currents is of great interest concerning micropower applications, bipolar. VLSI devices, and parasitic effects modeling. The nonideal component of base current sets the limit for transistor operation at very low currents, and its origin is still under controversy. Most of the published works refer to room-temperature measurements and they treat forward and reverse $B-E$ junction behavior separately. There is general agreement that reverse $I_{B}-V_{B E}$ characteristics diverge from ideal Schockley theory due to metallic precipitates that cause a field-dependent generation-recombination mechanism [1], [2]. However, for

Manuscript received September 27, 1983; revised May 15, 1984. This work was supported by U.S.-Spain Cooperative Project 793031.

C. Gonzalez-Bris was with the Departamento de Electronica, ETSI Telecomunicacion; Universidad Politecnica de Madrid, Ciudad Universitaria, Madrid-3, Spain. He is now with the Integrated Circuits Laboratory, Stanford University, Stanford, CA.

E. Muñoz is with the Departamento de Electronica, ETSI Telecommunicacion, Universidad Politecnica de Madrid, Ciudad Universitaria, Madrid-3, Spain. 